\title{
Threshold effects for two pathogens spreading on a network
}

\author{
M. E. J. Newman \\ Department of Physics and Center for the Study of Complex Systems, \\ University of Michigan, Ann Arbor, MI 48109-1120 and \\ Santa Fe Institute, 1399 Hyde Park Road, Santa Fe, NM 87501
}

\begin{abstract}
Diseases spread through host populations over the networks of contacts between individuals, and a number of results about this process have been derived in recent years by exploiting connections between epidemic processes and bond percolation on networks. Here we investigate the case of two pathogens in a single population, which has been the subject of recent interest among epidemiologists. We demonstrate that two pathogens competing for the same hosts can both spread through a population only for intermediate values of the bond occupation probability that lie above the classic epidemic threshold and below a second higher value, which we call the coexistence threshold, corresponding to a distinct topological phase transition in networked systems.
\end{abstract}

Social, technological, and biological networks of various kinds have been the subject of a large number of recent studies published in the physics literature [1, 2, 3, 4]. One of the principle practical applications of this body of work has been in modeling the spread of epidemic disease. Diseases spread over the networks of physical contacts between individuals $[5,6,7,8]$ and an understanding of the structure of these networks and the dynamics of disease upon them is crucial to the development of strategies for disease control. As it turns out, a large class of epidemic processes can be mapped onto bond percolation models [5, 8, 9, 10], allowing familiar techniques from statistical physics to be applied directly to their solution.

An issue of some interest in current epidemiological research is the behavior of competing pathogens $[11,12,13$, 14]. Two diseases may compete for the same population of hosts because one disease kills hosts before the other can infect them. Or there may be cross-immunity between the diseases such that exposure to one disease leaves the host alive but immune to further infection by either disease. Competing strains of influenza can show this type of behavior, for instance [13, 14]. The dynamics of competition between pathogens is in general complex, depending in particular on whether one pathogen gets a head start on the other in the population. In this paper we study the case in which two pathogens pass through the population at well separated intervals: one infects the population and causes an epidemic, leaving some fraction of the population immune or dead, and at some later time the second pathogen passes through the remaining population. (Our arguments could also be applied to two successive outbreaks of the same disease.) The question we address is if and when the second disease is able to spread. If a sufficient number of hosts is removed from the population by the first disease then spread of the second becomes impossible. As we will see there is a threshold value of the bond occupation probability or "transmissibility" for the first disease (a measure of contagiousness) at which this happens. This "coexistence threshold" coincides with a continuous phase transition similar to the well known epidemic transition, but the two transitions are quite distinct: the coexistence threshold is an additional property of the network topology.
Spread of both pathogens can occur only in the intermediate regime between the epidemic and coexistence thresholds. Among other things, we determine by exact analytic calculation for a broad class of networks the position of the two thresholds. For the much-studied case of a "scale-free" network, we find that while the epidemic threshold for the first disease is always zero, the coexistence threshold is not. A corollary of this result is that while a single disease on a scale-free network cannot be eradicated solely by lowering the transmissibility, a similar intervention in the case of two competing diseases can eradicate one of the diseases, but not both.

Consider then an epidemic taking place on a network of contacts between individuals. The network is represented by a graph in which vertices are individuals and (undirected) edges are contacts. The epidemic begins with a single individual and spreads along the contacts. Not every contact necessarily results in disease transmission however. We assume a generalized susceptible/infective/removed (SIR) dynamics for the disease of the kind described in [8] in which the disease spreads over edges with a probability $T$ called the transmissibility of the disease. This dynamics can be mapped onto a bond percolation process on the same graph with bond occupation probability equal to the transmissibility $[5,8,0,10]$. The connected clusters of vertices in the percolation process then correspond to the groups of individuals who would be infected by a disease outbreak starting with any individual within that cluster. Typically, for small values of $T$ there are only small clusters and hence only small disease outbreaks. But above some critical transmissibility $T_{c}$ an extensive spanning cluster or "giant component" appears, corresponding to an epidemic of the disease: once such a giant component is present, the pathogen reaching any of its members will infect them all and thereby reach an extensive fraction of the population. The value of $T$ at which the giant component first forms is called the epidemic threshold and it corresponds precisely to the percolation threshold for percolation on the contact network.

To be concrete, we examine in this paper the class of graphs that have specified degree distribution but which are otherwise random, in the limit of large graph size. (Recall that the "degree" of a vertex in a network is the number of edges 
connected to that vertex.) Such graphs have been studied in the past by many authors [15, 16, 17, 18, 19] and have become a standard arena for the exploration of epidemiological processes [7, 8]. Epidemiological processes have also been studied on other types of networks, such as networks with degree correlations [20, 21], and it seems likely that the results presented in this paper could be generalized to such cases, although we do not do that here.

Let $p_{k}$ be the fraction of vertices in our network that have degree $k$. We can also consider $p_{k}$ to be the probability that a randomly chosen vertex has degree $k$. The vertex at either end of a randomly chosen edge, on the other hand, has degree $k$ with probability proportional not to $p_{k}$ but to $k p_{k}$, the reason being that there are $k$ times as many edges connected to a vertex of degree $k$ than to a vertex of degree 1, and hence the probability that our edge will be one of them is also multiplied by $k$. We will primarily be interested in the distribution of the number of edges emerging from such a vertex other than the one we followed to get there. This excess degree is one less than the total degree of the vertex and therefore has a (correctly normalized) distribution

$$
q_{k}=\frac{(k+1) p_{k+1}}{\sum_{k} k p_{k}}=\frac{(k+1) p_{k+1}}{z},
$$

where $z=\langle k\rangle=\sum_{k} k p_{k}$ is the mean degree of the vertices in the network.

Our first pathogen can spread across the network if its basic reproductive number $R_{0}$ is greater than unity, i.e., if for every person infected the mean number of additional people they infect is greater than 1 .

When the disease arrives at a vertex, it has the chance to spread to any of the $k$ other neighbors of that vertex, each of which chances is realized with probability $T$ for an expected $T k$ additional vertices infected. Averaging over the distribution $q_{k}$ of $k$, we find that the basic reproductive number is

$$
R_{0}=T \sum_{k=0}^{\infty} k q_{k}=\frac{T}{\langle k\rangle} \sum_{k=0}^{\infty} k(k+1) p_{k+1}=T \frac{\left\langle k^{2}\right\rangle-\langle k\rangle}{\langle k\rangle} .
$$

Thus the disease spreads if and only if $T$ is greater than the critical value

$$
T_{c}=\frac{\langle k\rangle}{\left\langle k^{2}\right\rangle-\langle k\rangle} .
$$

To calculate the size of the epidemic when it does occur, it is convenient, following our previous approach [8, 17, 22], to define two probability generating functions for the distributions $p_{k}$ and $q_{k}$ :

$$
F_{0}(x)=\sum_{k=0}^{\infty} p_{k} x^{k}, \quad F_{1}(x)=\sum_{k=0}^{\infty} q_{k} x^{k}=\frac{F_{0}^{\prime}(x)}{z},
$$

where $F_{0}^{\prime}$ denotes the first derivative of $F_{0}$ with respect to its argument. In terms of these functions, for example, Eq. (3) can be written

$$
T_{c}=\frac{1}{F_{1}^{\prime}(1)}
$$

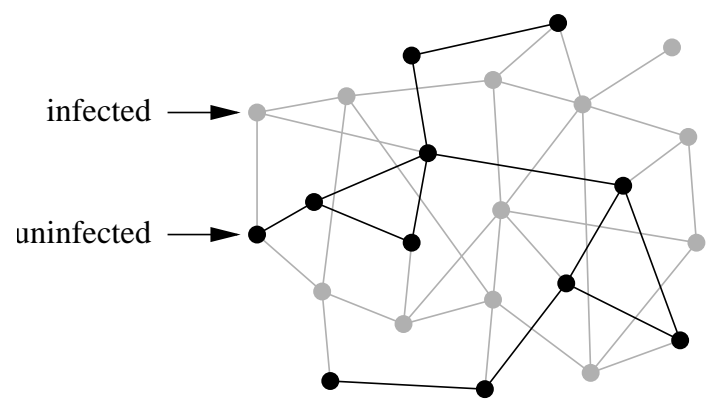

FIG. 1: An epidemic of the first disease spreads through the network leaving some fraction of the vertices either immune to further infection or dead, which we represent by simply removing them and their adjacent edges from the network (gray). The question we address is whether the remaining "residual network" (black) percolates and can therefore support the spread of the second disease.

Now let $u$ be the mean probability that a vertex is not infected by a specified neighboring vertex in the network during an epidemic outbreak of our disease. This quantity is equal to the probability that no transmission occurred between the two vertices, which is $1-T$, plus the probability that there was contact sufficient for transmission but that the neighboring vertex itself wasn't infected. The probability that the neighboring vertex wasn't infected is equal to the probability that it, in turn, failed to contract the infection from any of its $k$ other neighbors, which is just $u^{k}$ with $k$ distributed according to $q_{k}$. Thus the mean probability of the neighbor being uninfected is $\sum_{k=0}^{\infty} q_{k} u^{k}=F_{1}(u)$. Hence, $u$ must satisfy the equation

$$
u=1-T+T F_{1}(u) .
$$

Then the probability that a randomly chosen vertex is not infected is $\sum_{k=0}^{\infty} p_{k} u^{k}=F_{0}(u)$, and the fraction $S$ of vertices that do get infected is one minus this:

$$
S=1-F_{0}(u)
$$

Thus we can calculate the size of the epidemic by solving (6) for $u$ and substituting the result into (7). $S$ is also the probability of an epidemic occurring if the disease starts with a randomly chosen individual-with probability $1-S$ an outbreak fails to become an epidemic even when we are above the transition. If $S$ is regarded as an order parameter for the model, then the epidemic transition is a continuous phase transition in the mean-field universality class for percolation.

Now consider the case in which our first disease causes an epidemic in the network, leaving a fraction $S$ of vertices either dead or immune to infection by our second disease (or by a second wave of the first disease). To represent this mathematically, we remove these vertices from the network, leaving a smaller network of uninfected vertices which we call the residual graph-see Fig. 11 Only if this residual graph has a giant component will it be possible for the second pathogen, provided it has a suitably high transmissibility, to spread.

Clearly when $T=0$ for the first pathogen no individuals are infected and the entire graph remains for the second pathogen 
to exploit. Conversely, when $T=1$ an epidemic of the first pathogen will infect the entire giant component of the graph, and once this component is removed the second pathogen definitely cannot spread (since, in the limit of large size, random networks have only one giant component). In between these two extremes, we can expect a transition, which we now investigate. We begin by calculating the degree distribution of the residual graph. Once we have this distribution then, because the graph is uncorrelated, it is a straightforward exercise to determine whether it has a giant component or not.

Consider a vertex with degree $k$. Let $P$ (uninf., $m \mid k$ ) be the probability that it remains uninfected at the end of the first epidemic and has $m$ edges that are attached to other uninfected vertices. In other words, $P$ (uninf., $m \mid k)$ is the probability that this vertex belongs to the residual graph and has degree $m$ within that graph, given that it has degree $k$ in the graph as a whole.

This probability is equal to the probability that the vertex has $k-m$ unoccupied edges that attach to infected vertices and $m$ edges (occupied or not) that attach to uninfected vertices. The probability of an edge attaching to a uninfected vertex is just $F_{1}(u)$ and the probability of being unoccupied and attaching to an infected vertex is $(1-T)(1-$ $\left.F_{1}(u)\right)=u-F_{1}(u)$, where we have used Eq. (6). Then $P($ uninf., $m \mid k)=\left(\begin{array}{c}k \\ m\end{array}\right)\left[F_{1}(u)\right]^{m}\left[u-F_{1}(u)\right]^{k-m}$. Multiplying by the probability $p_{k}$ of having degree $k$ and summing over $k$ then gives the probability of being uninfected and having degree $m$ within the graph of uninfected vertices: $P($ uninf., $m)=$ $\sum_{k=m}^{\infty} p_{k}\left(\begin{array}{l}k \\ m\end{array}\right)\left[F_{1}(u)\right]^{m}\left[u-F_{1}(u)\right]^{k-m}$. Dividing by the prior probability $P($ uninf. $)=1-S=F_{0}(u)$ of being uninfected, the probability distribution of the degrees of vertices within the residual graph is

$$
P(m \mid \text { uninf. })=\frac{1}{F_{0}(u)} \sum_{k=m}^{\infty} p_{k}\left(\begin{array}{c}
k \\
m
\end{array}\right)\left[F_{1}(u)\right]^{m}\left[u-F_{1}(u)\right]^{k-m} .
$$

The generating function for this distribution is

$$
\begin{aligned}
G_{0}(x) & =\frac{1}{F_{0}(u)} \sum_{m=0}^{\infty} x^{m} \sum_{k=m}^{\infty} p_{k}\left(\begin{array}{c}
k \\
m
\end{array}\right)\left[F_{1}(u)\right]^{m}\left[u-F_{1}(u)\right]^{k-m} \\
& =\frac{1}{F_{0}(u)} \sum_{k=0}^{\infty} p_{k} \sum_{m=0}^{k}\left(\begin{array}{c}
k \\
m
\end{array}\right)\left[x F_{1}(u)\right]^{m}\left[u-F_{1}(u)\right]^{k-m} \\
& =\frac{1}{F_{0}(u)} \sum_{k=0}^{\infty} p_{k}\left[u+(x-1) F_{1}(u)\right]^{k} \\
& =\frac{F_{0}\left(u+(x-1) F_{1}(u)\right)}{F_{0}(u)} .
\end{aligned}
$$

Given this generating function, we can determine whether the residual network has a giant component using the method of Ref. [17]. We define

$$
G_{1}(x)=\frac{G_{0}^{\prime}(x)}{G_{0}^{\prime}(1)}=\frac{F_{1}\left(u+(x-1) F_{1}(u)\right)}{F_{1}(u)},
$$

which is the generating function for the excess degree of a vertex reached by following an edge in the residual graph, precisely analogous to Eq. (4). Then there is a giant component if and only if $G_{1}^{\prime}(1)>1$. Thus, the point $G_{1}^{\prime}(1)=1$ constitutes an additional phase transition in the system, other than the standard epidemic transition, at which a sufficiently contagious second pathogen can cause an epidemic after the passage of the first through the network. We call this the coexistence transition and the point at which it occurs the coexistence threshold. Making use of Eq. (10), we find that the transmissibility $T_{x}$ at this point is the solution of the equation

$$
F_{1}^{\prime}(u)=1,
$$

where $u$ is a function of $T$ via Eq. (6).

For instance, in the case of a Poisson degree distribution for the original network $p_{k}=\mathrm{e}^{-z} z^{k} / k$ ! (the standard Bernoulli random graph), we have $F_{0}(x)=F_{1}(x)=\mathrm{e}^{z(x-1)}$, which means that the normal epidemic threshold falls at $T_{c}=1 / z$ while the coexistence threshold falls at the point satisfying

$$
1=z F_{1}(u)=z(1-S) .
$$

If we can find $S$ from Eq. (7), it is then a straightforward matter to find $T_{x}$.

The size $C$ of the giant component in the residual graph, which sets an upper bound on the size of a possible second epidemic, is given by

$$
C=1-G_{0}(v), \quad v=G_{1}(v),
$$

as a fraction of the size of the residual graph [17]. To get the result as a fraction of the size of the original network, we then need to multiply by $1-S$. In Fig. 2 we show the sizes $S$ and $(1-S) C$ of the epidemic and the giant component on the residual graph as a function of transmissibility for the Poisson case. As the transmissibility increases from zero, the size of the residual giant component is initially equal to the size of the giant component of the entire graph, which is very nearly 1 . As $T$ passes the epidemic threshold for the first pathogen, however, the pathogen starts to spread and kills or renders immune to the second pathogen some fraction of the population, thereby reducing the size of the epidemic of the second pathogen. At some point—our coexistence threshold—so many are killed or made immune that too few are left to spread the second pathogen and $C$ reaches zero. Thus the epidemic spread of both pathogens is possible only in the intermediate regime of transmissibility $T_{c}<T<T_{x}$ indicated by the shaded area in the figure; if the transmissibility is either too low or too high, coexistence is impossible. In the inset of the figure we show how the two threshold values of the transmissibility, $T_{c}$ and $T_{x}$, vary as a function of mean degree for the Poisson case.

Of course, the mere existence of a giant component in the residual graph does not mean that the second pathogen will cause an epidemic. That depends on whether the transmissibility of the second pathogen is high enough. Repeating the analysis leading to Eq. (5), we find that the second pathogen can spread if its transmissibility is above the critical value $T_{c}^{\prime}=1 / G_{1}^{\prime}(1)$ or equivalently $T_{c}^{\prime}=1 / F_{1}^{\prime}(u)$-yet a 


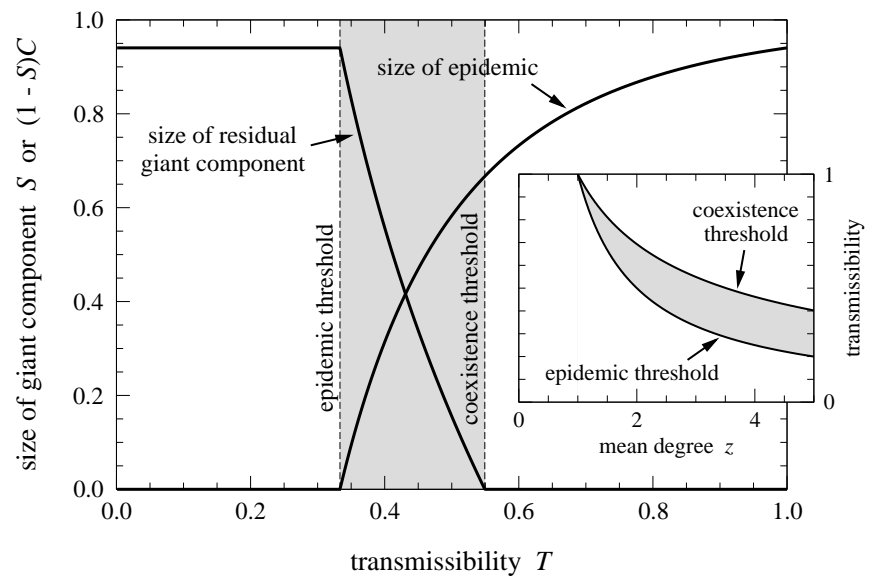

FIG. 2: The size of the epidemic of the first pathogen and the size of the residual giant component that it leaves behind, as a function of transmissibility on a graph with a Poisson degree distribution with mean degree $z=3$. Inset: the position of the two thresholds as a function of mean degree for the Poisson case. The shaded areas in the plots denote the region in which both pathogens can spread.

third threshold in our system (but one whose position is not solely a function of the network topology, since it depends also on the transmissibility of the first pathogen via Eq. (6). Noting that $F_{1}^{\prime}(x)$ is a polynomial with non-negative coefficients and therefore monotonic increasing on the positive real line (within its radius of convergence), and that $u \leq 1$ since it is a probability, we see that $F_{1}^{\prime}(u) \leq F_{1}^{\prime}(1)$, and hence that $T_{c}^{\prime} \geq T_{c}$ : the minimum transmissibility necessary for the second pathogen to spread is never less than that necessary for the first. This accords with our intuition: as we have shown elsewhere [8], vertices with high degree are more likely to be infected than those with low degree, and therefore we would expect the residual graph to have lower mean degree and hence higher epidemic threshold than the original network.

Another example of interest is that of a network with a power-law degree distribution $p_{k}=k^{-\alpha} / \zeta(\alpha)$, for some constant $\alpha$, where $\zeta(x)$ is the Riemann $\zeta$-function. Such networks are often called "scale-free." A variety of networks appear to be scale-free and they have attracted considerable attention in the recent literature [2, 3]. As is by now well understood [7, 23], (uncorrelated) scale-free networks with $\alpha<3$ have a vanishing epidemic threshold $T_{c}=0$ because the second moment $\left\langle k^{2}\right\rangle$ of the degree distribution in Eq. (3) diverges. Noting [17] that a power-law degree distribution gives a generating function $F_{0}(x)=\mathrm{Li}_{\alpha}(x) / \zeta(\alpha)$, where $\mathrm{Li}_{n}(x)$ is the $n$th polylogarithm of $x$, and applying Eq. 111), we find by contrast that the coexistence threshold in such a network is in general nonzero. Furthermore, the critical transmissibility for the second pathogen $T_{c}^{\prime}=1 / F_{1}^{\prime}(u)$ is also nonzero. (This nonzero threshold immediately implies that the residual network cannot itself be scale-free. The physical explanation of this result is that the first pathogen is more likely to infect higher-degree vertices and so selectively removes or immunizes the "hubs" in the network, destroying the power-law form. Removing hubs is well-known to be a good strategy for preventing the spread of disease [21, 24].)

The result $T_{c}=0$ implies that a single disease spreading on a scale-free network of this kind can never be eradicated by an intervention whose sole effect is to reduce the transmissibility. Our findings indicate, however, that for the case of two competing pathogens on such a network, one of them can be eradicated by an intervention that lowers the transmissibility, but not both.

To conclude, we have studied, using mappings to bond percolation, the problem of two diseases spreading through the network of contacts between members of a host population. We find that, in the case where hosts can be infected with either one or other, but not both, of the diseases, the spread of both is possible only for intermediate values of the transmissibility of the first disease. There are two phase transitions that mark the boundaries of this intermediate regime. The first is the standard epidemic transition below which the first disease is not contagious enough to spread at all; the second is an additional topological phase transition in the network that corresponds to the point at which the first disease removes from the population so large a fraction of the hosts that not enough remain to support the spread of the second disease.

We have here studied only the simplest case of competing pathogens. A number of variants of the problem are of interest. For instance, in some cases the first pathogen may confer upon those it infects only partial cross-immunity to the second, so that the probability of infection with the second pathogen is reduced but not entirely eliminated. This process could be modeled using an extension of the formalism described here in which the residual graph is formed by removing a fixed fraction, randomly selected, of the vertices affected by the first epidemic.

The author thanks Ben Kerr, James Koopman, Mercedes Pascual, and Carl Simon for useful conversations. This work was funded in part by the National Science Foundation under grant number DMS-0405348.

[1] S. H. Strogatz, Exploring complex networks. Nature 410, 268276 (2001).

[2] R. Albert and A.-L. Barabási, Statistical mechanics of complex networks. Rev. Mod. Phys. 74, 47-97 (2002).

[3] S. N. Dorogovtsev and J. F. F. Mendes, Evolution of networks. Advances in Physics 51, 1079-1187 (2002).

[4] M. E. J. Newman, The structure and function of complex networks. SIAM Review 45, 167-256 (2003).

[5] D. Mollison, Spatial contact models for ecological and epidemic spread. Journal of the Royal Statistical Society B 39, 283-326 (1977).

[6] L. Sattenspiel and C. P. Simon, The spread and persistence of infectious diseases in structured populations. Math. Biosci. 90, 341-366 (1988).

[7] R. Pastor-Satorras and A. Vespignani, Epidemic spreading in scale-free networks. Phys. Rev. Lett. 86, 3200-3203 (2001).

[8] M. E. J. Newman, Spread of epidemic disease on networks. 
Phys. Rev. E 66, 016128 (2002)

[9] L. M. Sander, C. P. Warren, I. Sokolov, C. Simon, and J. Koopman, Percolation on disordered networks as a model for epidemics. Math. Biosci. 180, 293-305 (2002).

[10] P. Grassberger, On the critical behavior of the general epidemic process and dynamical percolation. Math. Biosci. 63, 157-172 (1982).

[11] K. Dietz, Epidemiologic interference of virus populations. $J$. Math. Biol. 8, 291-300 (1979).

[12] C. Castillo-Chavez, W. Huang, and J. Li, Competitive exclusion in gonorrhea models and other sexually-transmitted diseases. SIAM J. Appl. Math. 56, 494-508 (1996).

[13] V. Andreasen, J. Lin, and S. A. Levin, The dynamics of cocirculating influenza strains conferring partial cross-immunity. $J$. Math. Biol. 35, 825-842 (1997).

[14] S. Gupta, N. Ferguson, and R. Anderson, Chaos, persistence, and evolution of strain structure in antigenically diverse infectious agents. Science 280, 912-915 (1998).

[15] T. Łuczak, Sparse random graphs with a given degree sequence. In A. M. Frieze and T. Łuczak (eds.), Proceedings of the Symposium on Random Graphs, Poznań 1989, pp. 165-182, John Wiley, New York (1992).

[16] M. Molloy and B. Reed, A critical point for random graphs with a given degree sequence. Random Structures and Algorithms 6 ,
161-179 (1995).

[17] M. E. J. Newman, S. H. Strogatz, and D. J. Watts, Random graphs with arbitrary degree distributions and their applications. Phys. Rev. E 64, 026118 (2001).

[18] F. Chung and L. Lu, The average distances in random graphs with given expected degrees. Proc. Natl. Acad. Sci. USA 99, 15879-15882 (2002).

[19] B. Bollobás and O. Riordan, The diameter of a scale-free random graph. Combinatorica 24, 5-34 (2004).

[20] M. Boguñá and R. Pastor-Satorras, Epidemic spreading in correlated complex networks. Phys. Rev. E 66, 047104 (2002).

[21] R. Cohen, S. Havlin, and D. ben-Avraham, Efficient immunization strategies for computer networks and populations. Phys. Rev. Lett. 91, 247901 (2003).

[22] D. S. Callaway, M. E. J. Newman, S. H. Strogatz, and D. J. Watts, Network robustness and fragility: Percolation on random graphs. Phys. Rev. Lett. 85, 5468-5471 (2000).

[23] R. Cohen, K. Erez, D. ben-Avraham, and S. Havlin, Resilience of the Internet to random breakdowns. Phys. Rev. Lett. 85, 4626-4628 (2000).

[24] Z. Dezsố and A.-L. Barabási, Halting viruses in scale-free networks. Phys. Rev. E 65, 055103 (2002). 